majeure partie purement et simplement des données que le receveur ne peut utiliser que rarement pour s'orienter dans son environnement. De là, il est impossible que les feuilles paroissiales réussissent à établir la liaison entre l'Eglise locale et les catholiques nonpratiquants. Une nouvelle orientation de la conscience communicative des rédacteurs de feuilles paroissiales et des responsables écclésiastiques serait nécessaire pour rendre la presse paroissiale plus effective dans le domaine publicistique. La condition primordiale à cela est une formation et une formation complémentaire dans cette direction au cours de laquelle les fondements de la science de la publicistique et de celle de la communication, de même que du travail journalistique devraient être donnés.

\title{
RESUMEN
}

La prensa parroquial austriaca apenas está en condiciones de funcionar como instrumento de comunicación entre la Iglesia local y la comunidad parroquial. Ello se debe sobre todo a deficientes conceptos redaccionales. El contenido de las Hojas Parroquiales apenas corresponde a las necesidades de información de los miembros de la parroquia. En su mayor parte publican simples datos, que solo raras veces pueden servir de orientación a los destinatarios. Por ello las Hojas Parroquiales tampoco pueden lograr el crear comunicación entre la Iglesia local y los católicos no practicantes. Para conseguir una prensa parroquial publicísticamente más efectiva, sería necesaria una nueva mentalidad respecto de la comunicación social en los redactores parroquiales y en los responsables eclesiásticos. Condición previa para ello es una formación y perfeccionamiento que proporcionen las bases de la ciencia publicista y de la comunicación social, así como las del trabajo periodístico.

\section{Glaubwürdigkeit bei anderen: eine kritische Stellungnahme}

\author{
von Robert W. Kraay
}

Die Gedanken und Vorschläge, die Wilhelm Gössmann in seinem Beitrag „Der Sprachgebrauch in der Kirche - kritisch $^{\text {"1 }}$ vorlegt, betreffen grundlegende Fragen. Je mehr man sich mit ihnen beschäftigt, desto klarer wird die Bedeutung der Glaubwürdigkeit. Gössmann gebraucht den Begriff Glaubwürdigkeit, um den Sprachgebrauch in der Kirche zu kritisieren. Für ihn entsteht Glaubwürdigkeit nicht aus dem Gebrauch bestimmter „richtiger" Wörter, sondern ist vielmehr gegründet auf persönliche Lebenserfahrung. Eine glaubwürdige Botschaft verbindet persönliche Erfahrung mit klarer sprachlicher Form. Unglaubwürdigkeit kann ihren Grund haben sowohl in der Unfähigkeit, sich auszudrücken, als auch im Mißbrauch sprachlicher Möglichkeiten, der einen falschen Anschein von Glaubwürdigkeit erzeugt. ${ }^{1 *}$

Robert W. Kraay hat die School of Journalism der Universität von Iowa absolviert und steht im Begriff, seine Studien mit dem Schwerpunkt, religiöse Publizistik' in Europa (Universität Salzburg) fortzusetzen. 
Jemand der seine eigenen Erfahrungen aufgibt und sich nicht mehr an ihnen orientiert, wird nicht nur anderen gegenüber unglaubwürdig, sondern ist auch sidh selbst gegenüber unwahr. Glaubwürdigkeit sich selbst gegenüber wird gewonnen dadurch, daß jemand sich selbst kennenlernt, und durch Meditation, die ihn zu einer Wahrheit führt, die er als für sich gültig erkennt. Glaubwürdigkeit bei anderen entsteht nicht dadurch, daß man Dinge sagt, die den anderen gefallen, sondern vielmehr dadurch, daß man sie ernst nimmt, selbst bei Meinungsunterschieden, daß man Respekt und gemeinsames Bemühen zeigt, die das wechselseitige Gespräd fördern. ${ }^{2}$ So entsteht ein Dialog, in dem die isolierte Sprache des "Ich" zu einem brüderlichen "Wir" wird. Die gemeinsame Verständnisbereitschaft, die daraus erwächst, wird ihrerseits zu einer Hilfe für die Sprache des Individuums. ${ }^{3}$

Eine Untersuchung des Sprachgebrauchs von Christen führt zu dem Eindruck, daß Unglaubwürdigkeit allgegenwärtig ist. Anderen gegenüber unglaubwürdig sein ist deshalb besonders gefährlich, weil diejenigen, die unglaubwürdig sind, sich darüber gewöhnlich keine Rechenschaft geben. Es scheint so zu sein, daß Leute dann besonders von sich überzeugt sind, wenn ihnen Glaubwürdigkeit am meisten fehlt.

Das bringt uns zu einem anderen Problem. Wenn Glaubwürdigkeit notwendig ist und wenn die ausgefahrenen und abgenützten Wege sprachlichen Ausdrucks aufgegeben werden sollen, wie kann man dann jemanden dazu bringen, jene zusätzliche Anstrengung der Meditation auf sich zu nehmen, die oben als Lösung zu ihrem Problem genannt wurde. Diese Menschen sind an ihren gewohnten Sprachschatz gebunden. Sie müssen durch den Gebrauch dieses Sprachschatzes zu ihrem Glauben kommen. Sie glauben, daß, wenn diese ihre Sprache für sie selbst gut genug ist, sie auch für andere gut genug sein müsse; sie glauben, daß in dieser Sprache Vertrauen enthalten sei. Wenn sogar die Bibel (oder kirchliche Dokumente) diese Sprache gebrauchen, wie könnte sie falsch sein? Jemand, der die richtigen Worte gebraucht, muß mit Sicherheit ein Glaubender sein; jemand, der das nicht tut, kann es auf keinen Fall sein. Diese Haltung ist weitverbreitet und muß mit großem Feingefühl angegangen werden, bevor etwas dagegen gesetzt werden kann.

Aber dies ist nur das halbe Problem. Diese Leute sind nicht nur sich selbst, sondern auch anderen gegenüber unglaubwürdig. Diese "leeren Worte ${ }^{\alpha}$ bilden eine Trennwand $z$ wischen ihnen und jedem, der sich nicht mit diesen Worten identifizieren kann. Das führt nicht zu einem Mangel an Kommunikation (was noch halb so schlimm wäre), sondern zu einer Art von Kommunikation, die keinen gegenseitigen Austausch kennt und Mißtrauen und Mißverständnis fördert.

Um Gössmanns Gedanken für die Praxis nutzbar zu machen, ist es notwendig, die Menschen zu verstehen, die sich der Probleme der Unglaubwürdigkeit bewußt sein sollten, und die Gründe für ihren Sprachgebrauch zu begreifen. Sein Artikel trägt diesem Aspekt nicht voll Rechnung. Gut ist seine Darstellung der Gebiete, in denen Unglaubwürdigkeit recht offenkundig wird, die beschreibt, wie man sich selbst und anderen gegenüber glaubwürdig werden kann, und einige Vorteile der Glaubwürdigkeit aufweist. Die Untersuchung der Begriffe "Kommunikation" und „Ritual" kann zu einem besseren Verständnis personalen Engagements in Kommunikationsprozessen führen und Einstellungen in Bezug auf Glaubwürdigkeit klarstellen. Sie kann zudem Anregungen entwickeln, die für Leute nützlich sind, die die Glaubwürdigkeit einer Gruppe - wie der Kirche - erhalten möchten. 


\section{Kommunikation}

Die Untersuchung von Kommunikation kann auf sehr verschiedenen Wegen angegangen und auf eine Reihe von Aspekten dieses Phänomens konzentriert werden. Einer dieser Wege, den Gössmann in seinem Artikel geht, ist die Prüfung von Sprache und Sprachgebrauch. Das von ihm genannte Kriterium für Glaubwürdigkeit zeigt eines der wichtigsten Prinzipien beim Studium von Kommunikation: Kommunikation ist ein menschliches Problem. Der Mensch ist die Grundlage dieser Untersuchung, bei der Glaubwürdigkeit und Kommunikation von gleichem Belang sind. Beide haben nicht nur mit Wörtern zu tun, sondern auch mit menschlicher Erfahrung.

Immer klarer wird die Erkenntnis, daß unsere Sprache nicht isoliert ist, sondern in einem größeren Kontext entsteht und gebraucht wird. ${ }^{4}$ Dieser Kontext ist ein wesentlicher Teil der Kommunikationssituation, in der Sprache gebraucht wird. Die Mißachtung dieses Kontextes erzeugt Unglaubwürdigkeit. Bei der Untersuchung von Sprache und Sprachgebrauch gilt es, die personale und kulturelle Geschichte derjenigen, die Sprache gebrauchen, die Formen der Kommunikation, die sie gebrauchen können und normalerweise gebrauchen, das Umfeld, in dem Sprache gebraucht wird und dessen Bedeutung für die Menschen zu berücksichtigen. ${ }^{5}$ Dies führt zu einem zweiten Prinzip: Wörter und Sprache umfassen nicht die Totalität der Kommunikation. Menschen, Umfeld, Wortform usw. sind von größter Bedeutung, aber selbst wo Worte fehlen, kann Kommunikation stattfinden und findet sie statt. Wortlose Kommunikation kann sehr bedeutsam sein, etwa dann, wenn jemand einen anderen ignoriert.

Gössmann war dieses Prinzip wohl bewußt, wenn er seinen Artikel in drei Teile gliederte: Predigt, Gebet und Umgangssprache. Dies sind aber noch recht grobe Klassifizierungen für die Untersuchung der genannten Kriterien. Predigten in verschiedenen Kirchen und in derselben Kirche zu verschiedenen Zeiten sind jeweils andere Geschehnisse. Eine Predigt ist nicht einfach eine Predigt. Diese Vorstellung ist zu statisch für die Erfassung dessen, was dabei geschieht. Während die Predigt entsteht und vorgetragen wird, gibt es andauernd Veränderungen: nicht nur der Sprecher verändert sich, auch die Hörer (oder Nicht-Hörer) denken nach, schweifen ab, werden hungrig und erfahren zahlreiche andere physische und geistige Veränderungen. Alle diese Veränderungen sind miteinander verquickt und ereignen sich fortwährend. Man kann niemals eine Predigt "haben“, man muß den Prozeß von Geben und Empfangen durchlaufen. Auf die gleiche Weise ist auch ein, bei verschiedenen Gelegenheiten, wiederholtes Gebet nicht gleich. Die Motivationen wechseln. Von der Umgangssprache ist bekannt, daß sie schnell sich verändert, häufig so sehr, daß Wörterbücher selten Redensarten der Umgangssprache zu definieren versuchen: Kommunikation ist ein Prozeß und findet statt in einer Welt voller Prozesse. Man kann sie nicht festlegen, will man nicht den Prozeß völlig verlieren.

Es darf angenommen werden, daß das Erreichen von Glaubwürdigkeit allen hilft, doch die Kommunikationssituationen, in denen dies geschieht, sind sehr unterschiedlich. Diese Situationen stellen Forderungen, die vernichtend sein können für Menschen, die gewisse Erwartungen an Verhalten und Reaktion aufgebaut und verwendet haben, und nicht nur für diese Menschen selbst, sondern auch für andere. Nehmen Sie etwa das Beispiel eines Menschen, der seinen Glauben in einem traditionellen Satz ausdrückt. Diesen Satz hatte er gelernt. Er war sich nicht ganz darüber im klaren, was der Satz bedeutete, aber er klang gut, und alle anderen schienen seine 
Bedeutung zu verstehen. Diese Tatsache beunruhigte diesen Menschen, und ef begann, seine innere Erfahrung zu überprüfen, um neue Ausdrücke seines Glaubena zu schaffen. Dadurch gewann er Glaubwürdigkeit sich selbst gegenüber. Jedod seinen Freunden gegenüber, die den alten Satz als einen Glaubensmaßstab benutzten, erschien er weniger religiös. $\mathrm{Da}$ die traditionellen Worte für seine Freunde so große Bedeutung gewonnen hatten, verhinderte der gewohnte Gebrauch dieser Worte eine ernsthafte Diskussion. Seine Freunde konnten nicht verstehen, was mit ihm geschehen war. Sie etikettierten ihn einfach als "Abtrünnigen ". In dieser Lage war die einzige Möglichkeit, mit seinen Freunden zu diskutieren, den ursprünglichen Satz wieder zu verwenden. Hier gilt das Prinzip, daß Kommunikation auf Erwartungshaltungen aufbaut. Durch Wiederholung entwickeln sich Symbole und gewinnen Bedeutung. Durch Wiederholung verlieren sie aber auch an Bedeutung. Wie das Beispiel zeigt, geht es nicht um den Inhalt der Wörter, sondern vielmehr darum, was Menschen in einer bestimmten Situation aus den Wörtern machen. Wörter können niemals nur aus ihren beschreibenden Definitionen bestehen. Wörter sind nichts als Farbflecken auf einem Blatt oder Schallwellen, solange sie nicht von jemand interpretiert werden. Es ist bekannt, wie sehr die Interpretation eines Wortes von Person zu Person differieren kann. Dieser Gedanke weist auf den grundlegenden Unterschied hin zwischen Glaubwürdigkeit mit sich selbst und Glaubwürdigkeit bei anderen. Beim Bemühen um Glaubwürdigkeit für uns selbst brauchen wir uns nicht mit Fragen der Interpretation zu befassen, wohl ist das für die Glaubwürdigkeit bei anderen von entscheidender Bedeutung. Grundsätzlich entsteht Bedeutung nicht durch die Wörter und Symbole, die jemand aufnimmt, sondern vielmehr durch das, was jemand für wichtig hält.

Diese Prinzipien der Kommunikation werden unten bei der Glaubwürdigkeit bei anderen verwendet werden.

\section{Ritual}

Menschen nehmen an einem Ritual teil, wenn sie die Sprache der Kirche - glaubwürdig oder unglaubwürdig - gebrauchen. Diese Teilnahme an einem Ritual schafft Zwänge für ihr Handeln und berührt dadurch den Prozeß, bei anderen Glaubwürdigkeit zu gewinnen. Der Prozeß des Rituals verdient Beachtung, weil er ein für die Glaubwürdigkeit wichtiger Faktor ist.

Wie oben schon aufgezeigt, ist es gefährlich, durch den Gebrauch eines Wortes in einer Kommunikationssituation auf andere Situationen Rückschlüsse zu ziehen. Deshalb können Dinge mit ritualem Charakter, die Christen tun, nicht in einem Terminus zusammengefaßt werden. Vielmehr können die Parameter des Rituals gekennzeichnet und individuelle Anwendungen für die jeweilige Situation gefunden werden von denen, die daran teilnehmen. Ritual kann allgemein beschrieben werden als eine symbolische Handlung mit emotionalem Charakter, immer wieder von einer Gemeinschaft vollzogen gegenüber demjenigen, das für sie von größter Bedeutung ist. ${ }^{b}$ Symbolische Handlungen könnten im wesentlichen beschrieben werden als Handlungen, denen jemand Bedeutung beimißt. Der Begriff symbolische Handlung ist insofern nützlich, als er die Tatsache unterstreicht, daß Kommunikation wesentlich durch einfache Bewegung (ob beabsichtigt oder nicht) geschieht. Um symbolische Handlung zu werden, muß die Bewegung (oder ihr Fehlen) von jemand 
interpretiert werden. ${ }^{7}$ Wie beim Begriff Glaubwürdigkeit wird dadurch auch hier das Mißverständnis vermieden, daß Wörter mit Kommunikation gleichzusetzen seien. Wenn Kommunikation stattfinden soll, muß es auch gegenseitige Beziehungen $\mathrm{zwischen} \mathrm{Menschen} \mathrm{geben.} \mathrm{Diese} \mathrm{Beziehungen} \mathrm{sind} \mathrm{das} \mathrm{Fundament} \mathrm{für} \mathrm{Gemeinschaft}$ und Dialog. Im Ritual werden diese Beziehungen über die Zeiten hin aufrecht erhalten, und die symbolischen Handlungen werden wiederholt. Der Begriff "was von größter Bedeutung ist" bleibt dehnbar und kann von einer Kommunikationssituation zur anderen wechseln. Bei einem Gottesdienst kann das Wichtigste für jemanden sein, Gott zu verehren. Für seinen Nachbarn ist das Wichtigste vielleicht, seinen Nebenmann durch seine Frömmigkeit zu beeindrucken. Nach dem Gottesdienst mag das Wichtigste für beide sein, nach Hause zu gehen und gut zu essen. So können sie an einem Ritual teilnehmen, das sie nicht schon während, sondern erst nach dem Gottesdienst zusammenführt. ${ }^{8}$ Das „Wichtigste“ bedeutet Engagement, das deutlich wird in Einsatz und Emotion während dieser besonderen Kommunikationssituation.

Ritual kann negativ und positiv gesehen werden. Die negative Betrachtung sieht Bilder wiederholter, sinnloser Handlungen, die weiter ausgeführt werden, auch wenn ihr Sinn verloren gegangen ist - eine recht unglaubwürdige Situation. Die positive Betrachtung sieht eine Gemeinschaft von Menschen, die eine symbolische Handlung ausführen vor ihrem Gott, der dabei zugleich Zeuge und Partner ist. Die positive Betrachtung ist bei weitem nicht die gebräuchlichste. In einer einzigen Situation sind beide Sehweisen möglich. Insider mögen ein Ritual positiv sehen, während diejenigen, die es von außen betrachten, es als ein eher lächerliches, seltsames Ereignis empfinden, das durch etwas anderes Vernünftigeres, Praktischeres ersetzt werden sollte, das mehr ihren eigenen Ritualen gleicht.

Entstehen und Wert dieser Betrachtungsweisen können durch die Beschreibung von vier recht unterschiedlichen Phasen des Rituals weiter geklärt werden. Die erste ist der Ursprung des Rituals. Während dieser Phase schaffen die Menschen Bindungen, ihre Handlungen werden symbolisch und gewinnen emotionalen Eifer, und sie konzentrieren sich auf etwas, das für sie von größter Bedeutung ist. In der zweiten Phase wird die Nützlichkeit des Rituals deutlich. Jeder nimmt uneingeschränkt am Ritual teil, niemand stellt seinen Wert in Frage, jeder anerkennt innerlich seine Bedeutung. In der dritten Phase beginnt das Ritual zu zerfallen. Die meisten bleiben auf der Erfahrungsstufe der zweiten Phase stehen, einige aber nehmen an der Handlung des Rituals teil, ohne seinen Symbolgehalt ganz zu verstehen und ohne angesprochen zu sein von dem, das als wichtig angesehen wird. Vielleicht beteiligen sie sich nur, weil man das von ihnen erwartet. Vielleicht haben sie niemals eine Erfahrung gemacht, die es ihnen ermöglichte, den Symbolcharakter zu begreifen, der im Ritual gebraucht wird. So kennen sie wohl die Worte und Handlungen, können aber nicht voll am Ritual teilnehmen. Das gefühlsmäßige Engagement schwindet, und es beginnt eine Periode wachsender Enttäuschung über das Ritual. Die vierte Phase ist die des "toten" Rituals. Die Emotion im Symbolcharakter des Rituals wird nicht mehr erfahren. Wohl bestehen die Handlungen und Worte fort, gibt es noch die Aura des Heiligen, aber niemand weiß mehr, was sie bedeuten. In solch verhärteter Situation fühlt man sich wohl, abgeschirmt von jeder möglichen Veränderung. Am Ende dieser Phase jedoch gibt es nur zwei Möglichkeiten: die Vernichtung aller Reste des alten Rituals oder das Wachsen eines neuen, anderen Rituals. 
Man kann die vier Phasen des Rituals mit dem Begriff der Glaubwürdigkeit verbinden. Der Ursprung des Rituals entspricht der Meditation und dem Mühen, seine eigene Erfahrung durch symbolische Handlungen anderen verständlich $\mathrm{zu}$ machen. Das Ritual ist nützlich, wenn man für diejenigen glaubwürdig ist, die zur gleichen Gemeinschaft gehören. Für die Außenstehenden wäre er nicht notwendigerweise unglaubwürdig, aber er wäre auch nicht in enger Gemeinschaft mit ihnen; Außenstehende hätten in diesem Fall nur eine kleine Basis, von der her sie die Glaubwürdigkeit beurteilen könnten. Zerfall des Rituals ist dann gegeben, wenn das, was sich entwickelt hat, zwar noch für einige sinnvoll ist, wenn Erfahrungen noch wechselseitig verstanden werden und Glaubwürdigkeit untereinander vorhanden ist; für die anderen Teilnehmer aber ist der Sinn verloren. Letztere stehen in der Gefahr, ihre Glaubwürdigkeit zu verlieren, weil ihre Handlungen keinen Bezug mehr zu ihren Erfahrungen haben. Bei der Phase des toten Rituals besteht die Verbindung von Symbolcharakter und Ritual nicht mehr, und man bleibt einfach nur deshalb dabei, weil man nicht weggegangen ist. Diese Phase bedeutet totale Unglaubwürdigkeit.

\section{Glaubwürdigkeit bei anderen}

Die Sprache der christlichen Gemeinschaft ist ein äußerst wichtiger Bestandteil ihres Rituals. Tatsächlich hat ja eine Gemeinschaft nicht nur ein einziges Ritual, sondern derer viele, die von ihren Mitgliedern in unterschiedlichen Kommunikationssituationen vollzogen werden. Die Sprache, auf die sich Gössmann bezieht, ist kennzeichnend für viele Rituale, die im Leben der Kirche stattfinden. Jedoch sind nicht alle Rituale, die in diesem Zusammenhang praktiziert werden, kirchenbezogene Rituale und gebrauchen nicht unbedingt die Sprache der Kirche. Jemand kann in der Kirche sein, am Ritual eines Gottesdienstes teilnehmen, und unglaubwürdig sein; wenn er sich aber an seinen Nachbarn wendet und ihm etwas zuflüstert, nimmt er für eine kurze Zeit an einem anderen Ritual teil, gebraucht eine andere Sprache und ist dabei glaubwürdig. Deshalb sollte Gössmann nicht die Worte kritisieren, nicht einmal die Menschen, sondern die Kommunikationssituation insgesamt. In jeder Kommunikationssituation gilt es, die Art des spezifischen, gerade praktizierten Rituals zu verstehen und die Phase, in der dem einzelnen und der Gemeinschaft das Ritual erscheint.

Wenn man die Sprache einer bestimmten Kommunikationssituation durch die Bestimmung der eben dort gültigen Ritual-Phase versteht, ist es leichter, denjenigen einen $R a t z u$ geben, die sich bemühen, Unglaubwürdigkeit zu vermeiden.

In der Phase des Entstehens eines neuen Rituals und des Aufbaus von Glaubwürdigkeit ist Ermutigung angezeigt. Diejenigen, die ihre Erfahrungen in neuen und ungewohnten Symbolcharaktern ausdrücken möchten, erleben das Stadium quälender Selbstbefragung und Skepsis von seiten derer, die sie schätzen.

Wenn ein bestimmter Symbolcharakter mit der Erfahrung zu einer Einheit verwachsen ist, entwickelt sich eine Situation großer Befriedigung, und das nützliche Leben des Rituals beginnt. Hier ist der Ort für eine Warnung vor den Folgen möglicher Nachlässigkeit und Selbstgenügsamkeit. Nach solcher Leistung besteht die Versuchung, auszuruhen, der nachzugeben aber die Gefahr der Unglaubwürdigkeit mit sich bringen könnte. 
Die Dynamik dessen, was in dieser Phase durch Kommunikation geschieht, kann vielleicht klarmachen, warum es geschieht. Erinnern wir uns daran, daß Glaubwürdigkeit bei anderen durch ernsthafte Diskussion entsteht. Weder Kommunikation noch Glaubwürdigkeit können allein auf Worte oder bestimmte Handlungen zurückgeführt werden, sondern sind zu erklären aus der Art und Weise, in der die Betroffenen diese Worte und Handlungen interpretieren. Worte und Handlungen, die für den einen sinnvoll sind, brauchen es für den anderen nicht zu sein. Weiterhin bleiben diese Worte nicht einmal für die gleiche Person auf unabsehbare Zeit sinnvoll. Das ist deshalb so, weil Kommunikation auf Menschen beruht, nicht auf etwas Statischem wie Wörtern oder Sätzen. Personen ändern sich. Jeder unterscheidet sich von seinem Nachbarn. Erfahrungen wiederholen sich nicht in der gleichen Weise, und verschiedene Menschen verbinden verschiedene Erfahrungen mit verschiedenen Wörtern. Der Dialog verringert die Distanz zwischen den Gesprächspartnern, weil viele Facetten von Erfahrung gemeinsam erarbeitet und in einen gemeinsamen Wortschatz eingebracht werden können, der allen Teilnehmern hilfreich ist. Dies erzeugt Gemeinschaft und legt auch die Grundlage für ein sinnvolles Ritual.

Beim nächsten Treffen werden diese Menschen neue Erfahrungen gemacht und sich auch wieder etwas verändert haben. Was bei früheren Diskussionen erarbeitet wurde, ist schon überholt. Die damals gefundene gemeinsame Sprache kann die neuen Erfahrungen nicht mehr fassen. Die Gemeinschaft beginnt, sich aufzulösen.

Dieser Prozeß der Erneuerung durch Diskussion und der Desintegration durch Veränderung vollzieht sich fortwährend, unabhängig davon, ob er schnell oder langsam vor sich geht. Je nach Intensität der Beziehungen bemißt sich das Maß an Energie, das für die Erneuerung oder die schließliche Beendigung der Beziehungen notwendig ist.

Diese Überlegungen weisen auf den Prozeß hin, der den Zerfall des Rituals einleitet. An irgendeinem, nicht genau bestimmbaren Punkt dieses Prozesses erscheint jemand, der seine Erfahrung nicht mehr in zufriedenstellender Weise mit den Worten und Handlungen des Rituals in Ubereinstimmung bringen kann. Bei diesem Stadium gibt es folgende mögliche Alternative. Einmal kann er sich selbst vom Ritual ausschließen und versuchen, seine eigene Glaubwürdigkeit (und vielleicht ein neues Ritual) zu begründen. Zum anderen könnte man ihm helfen, ein neues Verständnis für den Symbolcharakter des Rituals zu gewinnen, das ihn instandsetzen würde, auch die Handlung als vollwertiges Mitglied der Gruppe mitzuvollziehen. Dieser zweite Weg kann nicht von außen aufgezwungen werden, sondern ist nur möglich, wenn der Betroffene in entsprechender Weise motiviert ist. Wenn etwa Kinder die Rituale und Symbolcharaktere in Gottesdiensten nicht verstehen, genügt es nicht, ihnen eine Katechismuslektion zu halten. Ausreichend wäre schon, daß die Kinder selbst sich um ein neues Verständnis und Wertschätzung der Symbole bemühen sowie der Erfahrungen, die in ihnen dargestellt werden. So könnten sie sich wieder voll in die Gemeinschaft integrieren. Bei der Erziehung $\mathrm{zu}$ christlichem Ritual ist wichtig, daran $\mathrm{zu}$ erinnern, daß es nicht genügt, das System intellektuell zu verstehen.

Wenn jemand sich nicht selbst vom Ritual ausschließt und sich auch nicht um neuerliche Integration bemüht, wird Unglaubwürdigkeit die notwendige Folge sein. Das führt zum toten Ritual. Da sind die Wörter dann alles, und der Mensch ist nichts. Die Kommunikation geht dennoch weiter - zwischen ihnen und ihrer Um- 
gebung. Sie finden es sehr leicht, andere unnachgiebig nach den Kriterien ihrer Wörter zu beurteilen. Während dies geschieht, werden Außenstehende, die sie betrachten, sie als törichte, in Gewohnheiten gefangene, Menschen bezeichnen. Menschen, die auf totem Ritual bestehen, brauchen nur noch Nachsicht, weil sie auf nichts hören werden. Sie besitzen die Wahrheit, und die wird sich nie ändern.

Diese Diskussion hat vor allem die Mechanik der "Glaubwürdigkeit bei anderen" unterstrichen. Glaubwürdigkeit bei anderen ist eine der Glaubwürdigkeit für sich selbst verwandte, jedoch unterschiedliche und schwierigere Frage. Die Unterschiede und Zwänge müssen aber noch weiter entwickelt werden. Ziel dieser Úberlegungen war nicht, Gössmanns ausgezeichneten Artikel zu schmälern, sondern weitere Uberlegungen $\mathrm{zu}$ den weitreichenden und schwierigen Problemen der Glaubwürdigkeit anzuregen. Ein weiteres dorniges Problem, das Beachtung verdient in der Diskussion um die Sprache der Kirche, ist die Natur des Glaubens und die Art seiner Bindung an unsere Sprache und Kommunikation schlechthin. Welche Unterschiede gibt es, wenn überhaupt, $z$ wischen religiöser und sozialer Kommunikation? Ist diese Dichotomie überhaupt von Nutzen? Diese Fragen haben Auswirkungen auf alle drei hier besprochenen Bereiche: Glaubwürdigkeit, Kommunikation und Ritual.

Der hier entwickelte Begriff von Kommunikation und die Einführung des Begriffes Ritual können einige Aspekte dieses komplexen Problems erhellen. Sie führen vielleicht zu einem besseren Verständnis der Situation und eröffnen Wege, sie in der Praxis besser zu meistern. Wenn man Theorie in Praxis übersetzen will, spürt man immer das Bedürfnis, Regeln aufzustellen. Bei den hier vorgeschlagenen Prinzipien in Bezug auf Menschen und Kommunikation werden jedoch von außen aufgezwungene Regeln immer zu Unglaubwürdigkeit führen. Deshalb kann es immer nur darum gehen, den Menschen die Dynamik dieser Prozesse mehr bewußt zu machen, so daß sie sie für ihre eigenen Kommunikationssituationen fruchtbar machen und zur Bewältigung ihrer Probleme benutzen, immer vorausgesetzt, sie sind bereit, Anstrengung auf sich zu nehmen.

\section{Anmerkungen:}

1. Wilhelm Gössmann: Der Sprachgebrauch in der Kirche - kritisch, in: „Communicatio Socialis* $3: 1970,14-15$.

1*. Im Folgenden werden die Begriffe Glaubwürdigkeit und Unglaubwürdigkeit in diesem Sinne verwendet.

2. Wilhelm Gössmann: a.a.O.

3. Wilhelm Gössmann: Glaubwürdigkeit im Sprachgebrauch, München 1970, S. 14-15.

4. Vgl. Dell Hymes: Toward Ethnographies of Communication: The Analysis of Communicative Events, in: Giglioli, Pier Paolo (Hrsg.): Language and Social Context, Middlesex, England, Penguin Books Ltd. 1972, S. 24 ff.

5. Hymes: Models of the Interaction of Language and Social Life, in: Gumperz, John J., and Hymes, Dell (Hrsg.): Directions in Sociolinguistics, New York: Holt, Rinehart and Winston Inc. 1972, S. 55.

6. Diese Bestimmung ist eine Synthese der Ritual-Definitionen einer Reihe von Theologen, Anthropologen, Soziologen und Philosophen. Zur weiteren Information s. Robert Kraay, Ritual: A Study of the Literature. Unveröffentlichte, dem Doktorandenseminar der School of Journalism, University of Iowa, vorgelegte Arbeit, 1972. Diese Bestimmung von Ritual ist flexibler als jede andere. Mircea Eliade etwa sah das Ritual als "Nachahmung göttlicher Gesten oder bestimmter Episoden des heiligen Kosmos-Dramas die Legitimierung menschlicher Akte durch ein außermenschliches Modell ${ }^{\alpha}$ (in: Der 
Mythos der ewigen Wiederkehr). Peter Berger: The Sacred Canopy, Garden City, New York: Doubleday \& Company Inc. 1967, S. 26 andererseits sah das Ritual als ein Mittel, „das Heilige, das den gewöhnlichen Ereignissen des Lebens selbst beigemessen wird“, zu bekräftigen. Meine Vorstellung von dem "was wichtig ist" möchte - je nach Gesichtspunkt des Betroffenen - diese beiden Gedanken zusammenfassen. Wenn jemand das Heilige als ein außermenschliches Modell sieht, wird das für ihn wichtig sein. Wenn es um eine besondere Qualität geht, die den Ereignissen des Lebens selbst zugeordnet wird, dann wird das von Bedeutung sein. Auch alle weiteren Punkte in dieser Bestimmung haben eine gleiche Flexibilität.

7. Kenneth Burke gibt eine hilfreiche Beschreibung von symbolischer Aktion in „Dramatism", in "Interaction": International Encyclopedia of the Social Sciences, New York, Macmillan and Company 1966, Bd. II, S. 447.

8. Dieser Begriff dessen, "was von größter Wichtigkeit ist", soll als Paradox verstanden werden. Er bezieht sich sowohl auf das, was ein Mensch tut (menschliches Streben), als auf das, was vom Menschen unabhängig ist und ihn übersteigt.

\section{S U M M A R Y}

Following the publication of Wilhelm Gössmann's article „Der Sprachgebrauch in der Kirche - kritisch" (Language use of the Church - a critical approad) in no. 1, 1970 of "Comunicatio Socialis", the author discusses the credibility of Christians to distinguish between ritual and symbol. He proposes four phases which could be helpful in the clarification of the ritual from its origin. He discusses especially the mechanics of credibility with others. The author tries to further develop the contribution of Gössmann into a discussion on the nature of faith and its relation into our language and communication.

\section{RESUME}

Comme annexe ł l'article de Wilhelm Gössmann „L'usage de la langue dans l'Eglise critique“, paru en 1970 dans cette revue, l'auteur analyse la foi des chrétiens chez les autres surtout entre les deux pôles "rituel" et "caractère symbolique". Il distingue quatre phases dans le rituel, de son origine jusqu'a sa fin, qui lui semblent utile de mettre au clair. Il s'intéresse particulièrement pour la mécanique de la croyance chez les autres afin de poursuivre l'article de Gössmann; il aimerait animer la discussion sur la nature de la foi et la manière dont elle se lie à notre langue et à notre communication.

\section{RESUMEN}

Partiendo del trabajo de Wilhelm Gössmann "Der Sprachgebrauch in der Kirche - kritisch" (El empleo del lenguaje en la iglesia - críticamente), publicado en esta revista en 1970, el autor analiza la credibilidad de los cristianos entre los demás, sobre todo entre el "Ritual ${ }^{\alpha}$ y el "Carácter simbólico (Polonia). Distingue cuatro fases del Ritual desde su origen hasta su conclusión, fases que le resultan provechosas en orden al esclarecimiento. Se ocupa en especial de la mecánica de la credibilidad ante los demás para completar el estudio de Gössmann y quisiera dar impulso a la discursión en torno a la naturaleza de la fe y al modo de vincularse en nuestro idioma y comunicación. 